\title{
Modelling, Analysis and Design Considerations of Multi-Phase Bearingless Permanent Magnet Synchronous Machine
}

\author{
Alessandro Marfoli ${ }^{1}$, Mauro Di Nardo ${ }^{1}$, Seamus Garvey ${ }^{2}$, Michele Degano ${ }^{1}$, \\ Rajiv Vashisht ${ }^{2}$, Robert Turnbull ${ }^{2}$ and Chris Gerada ${ }^{1}$ \\ ${ }^{1}$ Department of Electrical Engineering, Power Electronics and Machine Control Group \\ ${ }^{2}$ Department of Mechanical, Materials \& Manufacturing Engineering \\ University of Nottingham, Nottingham, UK \\ alessandro.marfoli1@nottingham.ac.uk
}

\begin{abstract}
This paper presents a general multi-harmonics analytical model able to predict both torque and force behaviour of a multi-phase permanent magnet synchronous machine. This machine topology can be adopted in bearingless applications and in application where controlling the radial forces is deemed to be beneficial to reduce the system vibrations. The modelling approach is applied to a surface permanent magnet synchronous motor featuring a 6-phase symmetric winding configuration although the proposed model is general. The underlying mathematics behind the force and torque production is carefully described with emphasis on both assumptions and approximations. Finite element analysis are proposed for validation purpose of the proposed analytical model, thus highlighting benefits and limitations. Once validated, the model is used to draw general design guidelines for this kind of electrical machine highlighting the trade-off between the competitive needs of maximizing the torque and force capability while minimizing the interaction between force and torque production.

Index Terms-Analytical model, bearingless machine, multi-
\end{abstract} phase machine, radial forces, space vector decomposition.

\section{INTRODUCTION}

Electrical machines able to generate both torque and radial forces are usually used in bearingless applications, i.e. as a mean to remove one mechanical bearing. When used in tandem, it is possible to increase the number of controllable degrees of freedom (DOF) from 3 to 5, achieving full levitation capability (excluding the axial DOF) [1]. Another application of these electrical machine class is the active control of the system vibrations when the rotor is constrained by mechanical bearings. In both cases, their adoption allows pushing the maximum rotational speed which is often limited by the rotordynamic behaviour of the system (rotor, mechanical bearings, and housing structure). Initially (historic wise), electrical machines capable of producing both torque and radial forces have been designed integrating into one stator two different winding sets, one dedicated to the force production and the other to the torque [2]. Other design approaches proposed in literature to fulfill the same aim make use of bridge-configured winding or a couple of parallel 3-phases windings [3], [4]. The comparison exercise reported in [4] among these three winding configurations shows their respective benefits and drawbacks and concludes that there is not a clear winner. Neither configurations can be realized with as few hardware components as the traditional bearingless drive (two separate winding sets). The parallel winding configuration needs a more complex control to cater the intrinsic interaction between torque and force production while the bridge-configured one shows a higher fault tolerance. Another interesting approach to achieve at the same time torque and force production consists in the full exploitation of the increased electromagnetic degrees of freedom of multi-phase (i.e. more than 3) symmetrical winding [5]. Multi-three phase layout with sectorial distribution of each winding set have also been considered for applications demanding high reliability and fault tolerance [6]-[8], although shown pitfall in terms of control complexity. Any of these winding types have to be able to efficiently generate both torque and forces in a controllable way. This means minimizing the system complexity (both hardware and software), maximizing the torque and force to current ratios and minimize the interaction between these two outputs. In order to asses these aspects, it is of paramount importance to develop an analytical model of the electrical machine able to fully capture the complex electromagnetic behaviour.

The aim of this paper is to present a comprehensive analytical model applied to a multi-phases permanent magnet synchronous machine with the aim of highlighting the potentials and design challenges of this machine topology. After the analytical background introduction on how torque and radial force are generated in electrical machines, the analytical model is implemented and FE analysis are performed for validation purpose. Special emphasis will be placed on the design considerations which are often overlooked when considering bearingless applications. In particular, the trade-off between the competitive needs of maximizing the torque and force capability while minimizing the interaction between force and torque production is investigated via the analytical model [9], [10]. It will be shown that the magnetic load and the coil pitch of a six phases permanent magnet synchronous machine have the biggest impact on the machine performance and on the trade-off between torque and force requirements. 


\section{ANALYTICAL BACKGROUND}

In this section the main analytical equations to compute forces and torque of an ideal (linear magnetic behaviour of the iron materials) and isotropic electrical machine are recalled [11]. The analytical calculation of the electromagnetic torque $T(\alpha, \bar{i})$ is done derivating the co-energy with respect to the rotor position as reported in (1)

$$
T(\alpha, \bar{i})=\frac{\partial C_{E}(t)}{\partial \alpha}=\frac{\partial}{\partial \alpha}\left\{\frac{r_{g} l \delta}{2 \mu_{0}} \int_{0}^{2 \pi} B_{n}^{2}(\gamma, \alpha, \bar{i}) d \gamma\right\}
$$

where $B_{n}$ is the radial component of the airgap flux density, $\gamma$ is the tangential coordinate spanning $[0,2 \pi), \alpha$ the rotor position and $\bar{i}$ the set of currents flowing in the stator phases. The airgap flux density $B_{n}$ can be written as in (2) in terms of stator and rotor magneto motive forces: $M_{s}$ and $M_{r}$.

$$
B_{n}(\gamma, \alpha, \bar{i})=\frac{\mu_{0}}{\delta} \cdot\left[M_{s}(\gamma, \bar{i})+M_{r}(\gamma, \alpha)\right]
$$

In order to determine the torque, the expression $B_{n}^{2}$ needs to be calculated as in (8), where three terms can be noticed. The correlation between these three components and the torque are reported in (9). The integration along the coordinate $\gamma$ and the derivation with respect to $\alpha$ are fundamentally important to identify which interaction between stator and rotor m.m.f. leads to a non-null torque.

The first and third terms in (9) do not produce any torque component. In fact, the first one $M_{s}$ is $\alpha$ independent so the derivation with respect to $\alpha$ does produce zero torque. The third one $M_{r}$ depends on $\alpha$ but it produces zero torque since the integral of its squared value with respect to $\gamma$ is independent from $\alpha$. The second term in (8) is the only one that contribute to the torque; its expression is:

$$
T(\alpha, \bar{i})=\frac{r_{g} l \mu_{0}}{\delta} \int_{0}^{2 \pi} M_{s}(\gamma, \bar{i}) \frac{\partial M_{r}(\gamma, \alpha)}{\partial \alpha} d \gamma
$$

In order to identify the harmonic orders of magneto motive force that that interact each other generating torque, the two terms $M_{s}(\gamma, \bar{i})$ and $M_{r}(\gamma, \alpha)$ are written as summation of harmonics (10). Substituting (10) in (3), it is possible to solve the integral of the torque expression. The solution is reported in (11). As already well known, non-null torque components are produced by the interaction of stator and rotor magneto motive force harmonics only when $h-q=0$, where $h$ and $q$ are the harmonic orders of $M_{s}(\gamma, \bar{i})$ and $M_{r}(\gamma, \alpha)$, respectively. Synchronous harmonic components travelling at the same speed $\left({ }^{h} w t={ }^{q} w t\right)$ produce $\mathrm{DC}$ torque value while asynchronous harmonic components rotating at different speeds generate $\mathrm{AC}$ torque value, i.e. torque ripple. The final expression of a generic $\tilde{h}$-torque component is reported in (4) where $\tilde{h}=h=q$.

$$
T_{\tilde{h}}(\alpha, \bar{i})=\frac{\pi r_{g} l \mu_{0}}{\delta} \cdot{ }^{\tilde{h}} M_{s}(\bar{i})^{\tilde{h}} M_{r} \sin \left( \pm w t-{ }^{\tilde{h}} w t+{ }^{\tilde{h}} \gamma_{s r}\right)
$$

The radial forces produced by the stator currents can be computed applying the Maxwell stress tensor $\left(T_{M}\right)$ to the airgap flux density:

$$
T_{M}(\alpha, \bar{i})=\frac{1}{2} \cdot\left(H_{n} B_{n}-H_{t} B_{t}\right) \hat{n}+H_{t} B_{n} \hat{t}
$$

Although both radial $(n)$ and tangential $(t)$ components of flux density contribute to the Maxwell stress tensor, in many cases $H_{t} B_{t}$ terms can be neglected. Therefore, (5) can be simplified as in the following

$$
T_{M}(\gamma, \alpha, \bar{i})=\frac{B_{n}^{2}}{2 \mu_{0}} \hat{n}+\frac{B_{t} B_{n}}{\mu_{0}} d S \hat{t}
$$

The elementary force $d \bar{F}_{y}$ applied to the elementary rotor surface $d S$ can be be computed as follows

$$
d \bar{F}_{y}(\gamma, \alpha, \bar{i})=\bar{T}_{M}(\gamma, \alpha, \bar{i}) \cos (\gamma) d S
$$

A further simplification of the problem that enables a slender revision of the equations consists in neglecting the tangential component of the flux density $B_{t}$ and therefore also the

$$
\begin{aligned}
& B_{n}^{2}(\gamma, \alpha, \bar{i})=\left(\frac{\mu_{0}}{\delta}\right)^{2} \cdot\left[M_{s}^{2}(\gamma, \bar{i})+2 M_{s}(\gamma, \bar{i}) M_{r}(\gamma, \alpha)+M_{r}^{2}(\gamma, \alpha)\right] \\
& T(\alpha, \bar{i})=\frac{\partial}{\partial \alpha}\left\{\frac{r_{g} l \mu_{0}}{2 \delta} \int_{0}^{2 \pi}\left[M_{s}^{2}(\gamma, \bar{i})+2 M_{s}(\gamma, \bar{i}) M_{r}(\gamma, \alpha)+M_{r}^{2}(\gamma, \alpha)\right] d \gamma\right\} \\
& M_{s}(\gamma, \bar{i})=\sum_{\tilde{h}}{ }^{\tilde{h}} M_{s}(\bar{i}) \cdot \cos \left(p \gamma \tilde{h}+{ }^{\tilde{h}} \gamma_{s} \pm w t\right) \quad, \quad M_{r}(\gamma, \alpha)=\sum_{q=1}^{\infty}{ }^{q} M_{r} \cdot \cos \left(p \gamma q+{ }^{q} \gamma_{r}+{ }^{q} w t\right) \\
& \int_{0}^{2 \pi} M_{s}(\gamma, \bar{i}) \frac{\partial M_{r}(\gamma, \alpha)}{\partial \alpha} d \gamma=\int_{0}^{2 \pi} \sum_{h=1}^{\infty} \sum_{q=1}^{\infty} \frac{{ }^{h} M_{s}(\bar{i}) \cdot{ }^{q} M_{r}}{2} \\
& {\left[\sin \left(p \gamma(h+q)+{ }^{h} \gamma_{s}+{ }^{q} \gamma_{r} \pm w t+{ }^{q} w t\right)+\sin \left(p \gamma(h-q)+{ }^{h} \gamma_{s}-{ }^{q} \gamma_{r} \pm w t-{ }^{q} w t\right)\right] d \lambda} \\
& =\sum_{h=1}^{\infty} \sum_{q=1}^{\infty} \pi \cdot{ }^{h} M_{s}(\bar{i}) \cdot{ }^{q} M_{r}(\bar{i}) \sin \left({ }^{h} \gamma_{s}-{ }^{q} \gamma_{r} \pm w t-{ }^{q} w t\right) \\
&
\end{aligned}
$$




$$
\begin{aligned}
& F_{y}(\alpha, \bar{i})=\frac{r_{g} l \mu_{0}}{2 \delta^{2}} \int_{0}^{2 \pi}\left[M_{s}^{2}(\gamma, \bar{i})+2 M_{s}(\gamma, \bar{i}) M_{r}(\gamma, \alpha)+M_{r}^{2}(\gamma, \alpha)\right] \cos (\gamma) d \gamma \\
& \int_{0}^{2 \pi} M_{1}(\gamma, \bar{i}) M_{2}(\gamma, \bar{i}) \cos (\gamma) d \gamma=\int_{0}^{2 \pi} \sum_{\xi=1}^{\infty} \sum_{j=1}^{\infty} \frac{\xi^{\xi} M_{1}(\bar{i}) \cdot{ }^{j} M_{2}(\bar{i})}{4} \\
& {\left[\cos \left(p \gamma(\xi+j \pm 1)+{ }^{\xi} \gamma_{1}+{ }^{j} \gamma_{2}+{ }^{\xi} w t+{ }^{j} w t\right)+\cos \left(p \gamma(\xi-j \pm 1)+{ }^{\xi} \gamma_{1}-{ }^{j} \gamma_{2}+{ }^{\xi} w t-{ }^{j} w t\right)\right] d \lambda} \\
& =\sum_{\xi=1}^{\infty} \sum_{j=1}^{\infty} \pi \cdot \frac{\xi^{\xi} M_{1}(\bar{i}) \cdot{ }^{j} M_{2}(\bar{i})}{2} \cos \left({ }^{\xi} \gamma_{1}-{ }^{j} \gamma_{2}+{ }^{\xi} w t-{ }^{j} w t\right) \quad \text { if } \quad \boldsymbol{\xi}-\boldsymbol{j} \pm \mathbf{1}=\mathbf{0}
\end{aligned}
$$

second term in (6). By doing so, the expression of the yforce component $F_{y}$ applied to the rotor can be written as in (14) where the force is calculated by integrating (7) along the angular coordinate $\gamma$.

$$
F_{y}(\alpha, \bar{i})=\frac{r_{g} l}{2 \mu_{0}} \int_{0}^{2 \pi} B_{n}^{2}(\gamma, \alpha, \bar{i}) \cos (\gamma) d \gamma
$$

Similarly to the torque computation, the flux density expression $B_{n}^{2}(\gamma, \alpha, \bar{i})$ in (8) can be substituted in (14), thus giving the expression (12). In order to identify the force contribution of the three terms in (12), the generic resolution, valid for any of the three terms is reported in (13), where $M_{1}$ and $M_{2}$ could be either $M_{s}$ or $M_{r}$. It is evident that the three terms in (12) can potentially produce a radial force amplitude not null under the same general condition:

$$
\xi-j \pm 1=0
$$

In other words, the harmonic orders of the stator and rotor magneto motive forces have to differ with respect to each other of a unity in order to produce a non-null radial force.

Considering all the above formulations the following considerations can be drawn.

- The term $2 M_{s}(\gamma, \bar{i}) M_{r}(\gamma, \alpha)$ in (12) represents the most important contribution to the radial force production when considering permanent magnet synchronous machine and it constitutes the main controllable term. The expression of the y-force component for a generic set $\xi, j$ of harmonics is reported below

$$
F_{y_{\xi j}}(\alpha, \bar{i})=\frac{\pi r_{g} l \mu_{0}}{2 \delta^{2}} \cdot{ }^{\xi} M_{s}(\bar{i}) \cdot{ }^{j} M_{r} \cos \left({ }^{\xi} w t-{ }^{j} w t+\gamma_{s_{\xi j}}\right)
$$

- In order to produce both torque and radial forces at the same time $M_{s}$ must feature both odd and even harmonics. In the majority of the cases, the maximum harmonic of $M_{r}$ (usually having order $p$ equal to the pole pairs) is used to generate the main component of both torque and forces, therefore the term $M_{s}$ must exhibit both $p$ and $p \pm 1$ harmonic orders in order to produce both torque and force.

- When both torque and force are produced at the same time, the term $M_{s}^{2}(\gamma, \alpha, \bar{i}) \cos (\gamma)$ in (12) generates notnull radial force components. This terms is the cause of the coupling between force and torque production and it can be considered undesired.
- For the majority of the rotor structures $M_{r}$ exhibits an anti-symmetric characteristic, thus it features only odd harmonics. In this cases, no radial forces are produced by the term $M_{r}^{2}(\gamma, \alpha) \cos (\gamma)$ in (12). On the contrary, asymmetric rotor structures, such as consequent pole surface permanent magnet, produce radial force also via the term proportional to $M_{r}^{2}$.

It is important to underline that, when the fundamental harmonic of $M_{r}$ is used to generate both torque and forces, the contribution $M_{s}^{2}(\gamma, \alpha, \bar{i}) \cos (\gamma)$ behaves as a uncontrollable parasitic radial force component. At the design stage, this term should be minimized because it might cause the errors between the desired and the actual radial force. This phenomena will be described in details in the next sections.

Considering all the above, the simplest way to generate controllable radial forces is to have a motor featuring a winding arrangement able to produce independently at least two consecutive stator m.m.f. harmonic orders. Increasing the number of phases of a symmetrical winding layout is one of the option (unarguably the most elegant) allowing the independent production of multiple controllable magneto motive force harmonics.

\section{NUMERICAL IMPLEMENTATION AND FE VALIDATION}

In the next two subsections, the numerical implementation of the analytical formulation described in the previous section is reported along with its FE validation. The last subsection investigates the coupling between force and torque production and asses the validity of the analytical model over a wide operating conditions.

\section{A. Numerical implementation}

A numerical implementation of the analytical model based on winding function (WF) is reported in this section for a six-phase winding configuration featuring two rotor pole pairs, Fig.1. As a case study, a surface mounted permanent

TABLE I: Design assumptions.

\begin{tabular}{ccccccc}
\hline $\begin{array}{c}J_{\text {lin }} \\
{[k A / m]}\end{array}$ & $\begin{array}{c}J \\
{\left[A / m^{2}\right]}\end{array}$ & $\begin{array}{c}B_{\text {gap }} \\
{[T]}\end{array}$ & $\begin{array}{c}B_{\text {tooth }} \\
{[T]}\end{array}$ & $\begin{array}{c}B_{\text {yoke }} \\
{[T]}\end{array}$ & $\begin{array}{c}T \\
{[N m]}\end{array}$ & $\begin{array}{c}V \\
{[V]}\end{array}$ \\
\hline 12 & 4 & 1 & 1.4 & 1.4 & 1.5 & 150 \\
\hline
\end{tabular}




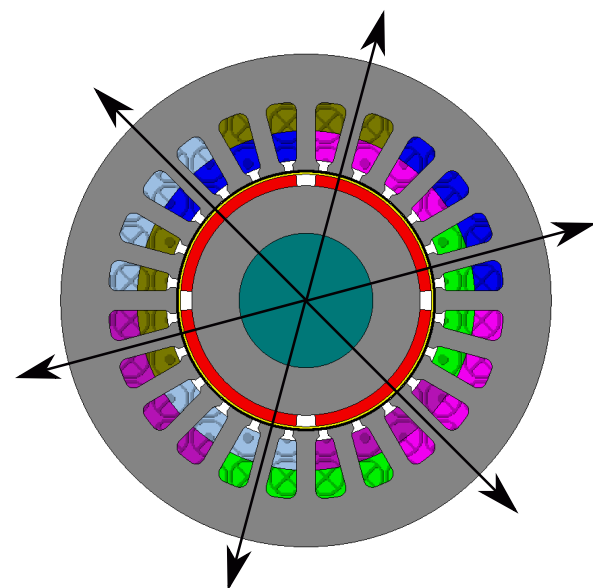

Fig. 1: Motor layout: FE model.

magnet motor is considered. The motor layout has been sized considering the assumptions reported in Tab. I.

The modelling approach adopted, considers only radial components of the airgap flux density and linear behaviour of soft magnetic materials. The symmetric winding arrangement of the hexaphase motor considered for the analysis is shown in Fig.1, where the black arrows represent the magnetic axes of the 6 phases. The related WFs representative of the motor winding layout are reported in Fig. 2a). The harmonic spectrum of one of the phases is also shown in Fig. 2b), where $1^{\text {st }}, 2^{\text {nd }}$ and $3^{\text {rd }}$ are the most significant components.

The matrix of inductances $\boldsymbol{L}_{\boldsymbol{p} \boldsymbol{h}}$ of such winding configuration, featuring magnetic axis of the phases equally spaced along the $2 \pi$ mechanical degrees assumes a circulating structure [12]. Such matrix can be diagonalized via the so-called space vector decomposition $(s v d)$, whose transformation matrix is computed as $\boldsymbol{T}=\boldsymbol{F} \boldsymbol{P}$, being $\boldsymbol{F}$ and $\boldsymbol{P}$ the Fourier and Park transformation matrixes, respectively.

The svd allows to independently control two space harmon-
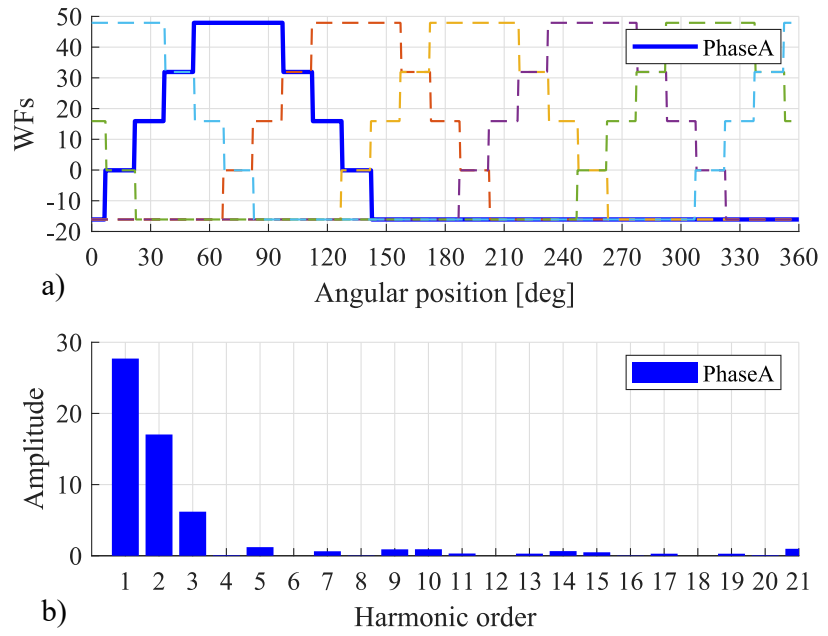

Fig. 2: a) WF profiles and b) harmonic spectrum. ics: the one having order $p=1$ to produce force and the order $p=2$ to produce torque. The transformed matrix of inductance

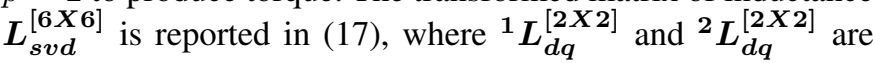
the inductance matrices of the first and second spaces while $\boldsymbol{L}_{\mathbf{0}}^{[\mathbf{2} X \mathbf{2}]}$ is the homopolar contribution.

$$
\boldsymbol{L}_{\boldsymbol{s v d}}=\boldsymbol{T}^{\boldsymbol{T}} L_{a b c} \boldsymbol{T}=\left(\begin{array}{ccc}
{ }^{1} \boldsymbol{L}_{\boldsymbol{d q}} & \ldots & 0 \\
\vdots & { }^{2} \boldsymbol{L}_{\boldsymbol{d q}} & \vdots \\
0 & \ldots & \boldsymbol{L}_{\mathbf{0}}
\end{array}\right)
$$

In order to improve the accuracy of the numerical implementation of the model, stator slot openings functions describing the airgap geometry are adopted as in [13]. The motor performance are computed supplying the stator windings of the hexphase motor in order to produce torque and a constant unidirectional force, while the rotor is rotating of a complete mechanical revolution.

In Fig. 3, the current waveforms when the motor generates the rated torque, the rated force and both of them are reported. It is worth to notice that the currents exhibit a sinusoidal profile in all the three operating conditions. To generate force (space $p=1$ is excited), the shift between the currents is $2 \pi / 6$ as shown in Fig. 3a). On the contrary, the shift between the currents of the different phases is $2 \pi / 3$ when the torque has to be produced, Fig. 3b).
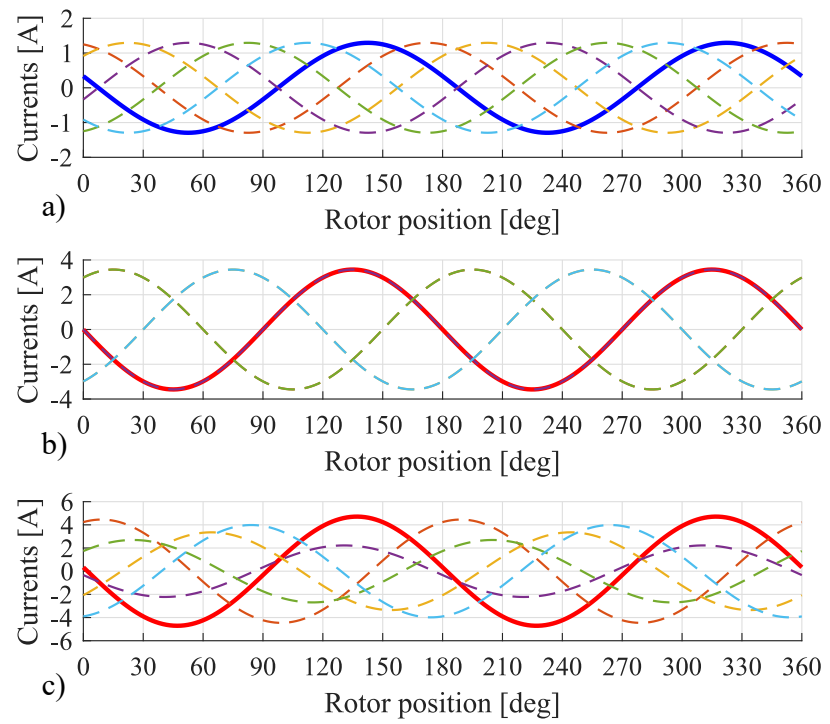

Fig. 3: Current waveforms: a) force generation, b) torque generation and c) both force and torque generation.

\section{B. FE validation}

Results obtained from the analytical model are compared against the FE results in Fig. 4 for validation purpose when producing both the rated torque and force. In Fig. 4a) and b) $\mathrm{x}-\mathrm{y}$ force components ( $\mathrm{Fx}$ and Fy) against rotor position and torque profile are reported. The torque profile exhibits a very good agreement against the FE results while the analytical predicted forces does not perfectly match the FE simulation. This discrepancy is due to the assumption of neglecting 

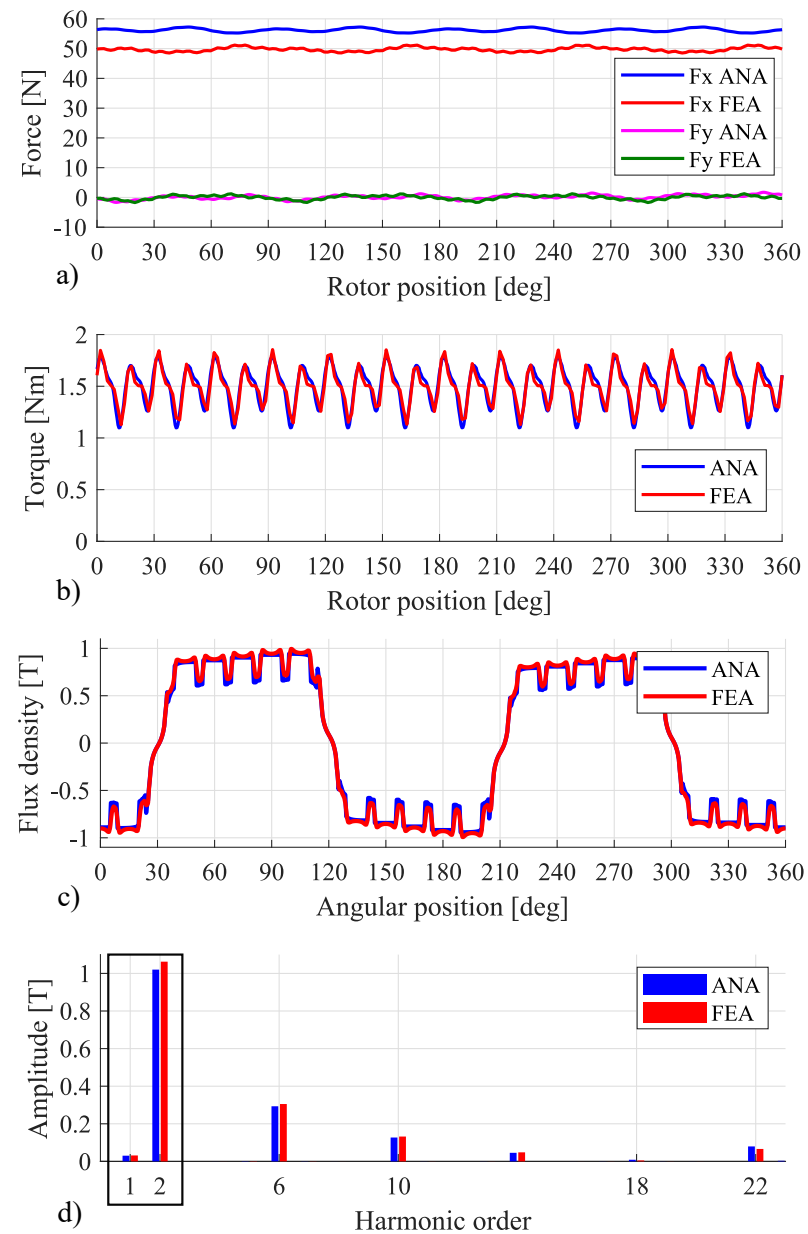

Fig. 4: Performance comparison between analytic (ANA) and FEA: a) Fx Fy waveforms, b) torque profile, c) airgap flux density and d) harmonic spectrum.

tangential component of flux density in the force computation. The influence of the tangential flux density components on the force production depends on the machine geometry and in particular on the mechanical airgap and magnet thickness. Such influence becomes more relevant as the overall magnetic airgap increases. Therefore, when evaluating machines with small airgap values, the analytical force estimation is reliable even with such a strong assumption.

Fig. 4c) and d) show the analytical computation of the radial airgap flux density (in one time instant) and its spectrum when the motor generates both torque and force. A very good agreement of the harmonic spectrum is achieved thanks to the implemented slot opening functions.

\section{Torque and force coupling}

After the preliminary validation reported above, the numerical model has been further compared against FE analysis to evaluate its capability of predicting force and torque and quantify the coupling effects between these two output quantities.

The coupling coefficient $K C$ defined in (18), represents the deviation of the force with respect to the reference when torque and force are produced at the same time. The space current phasors of the first (to produce force) and second order (to produce torque) can be defined as $\dot{i}_{p 1}$ and $i_{p 2}$, respectively.

$$
K C=\max _{n}\left\{\frac{\left\|F_{x y, i}-\left.F_{x y}\right|_{i_{p 2=0}}\right\|}{\left.F_{x y}\right|_{i_{p 2}=0}}\right\} \quad i=1, \ldots, n
$$

In (18), $\left.F_{x y}\right|_{i_{p 2}=0}$ represents the radial force amplitude when zero torque is generated $\left(i_{p 2}=0\right)$, while $n$ is the number of operating conditions featuring same force amplitude and direction but different torque value. According to the definition of coupling coefficient, no coupling effect occurs when $K C=0$.

In the following, two different operating conditions are examined in order to investigate such coupling phenomena. Indeed, when producing different average forces in terms of module and directions by playing on the vector $i_{p 1}$, several torque values can be produced acting either on the module or the phase of the current vector $i_{p 2}$.

Fig.5 and Fig. 6 show the steady state average force in the $\mathrm{x}-\mathrm{y}$ plane computed when considering two force amplitudes (two $\left|i_{p 1}\right|$ values) and different torque values acting on the phase of $i_{p 2}$ (Fig.5) and on the module of $i_{p 2}$ (Fig.6). The black markers (diamond) in these two figures represent the average force when producing zero torque.

The comparison between the analytical and FE analysis confirms that the developed analytical model is able to give a fair representation of the motor performance over a wide operating conditions. Indeed, the force prediction exhibit a maximum error of $18 \%$ in all the considered operating points.

Fig.5 shows that for each value of $i_{p 1}$ (different force direction and amplitude), the radial force generated by the motor change in relation with the advance angle $\angle \dot{i}_{p 2} \mid$ of the current vector responsible for the torque production $(0,90$, 180,270 [deg] are considered in the showed results).

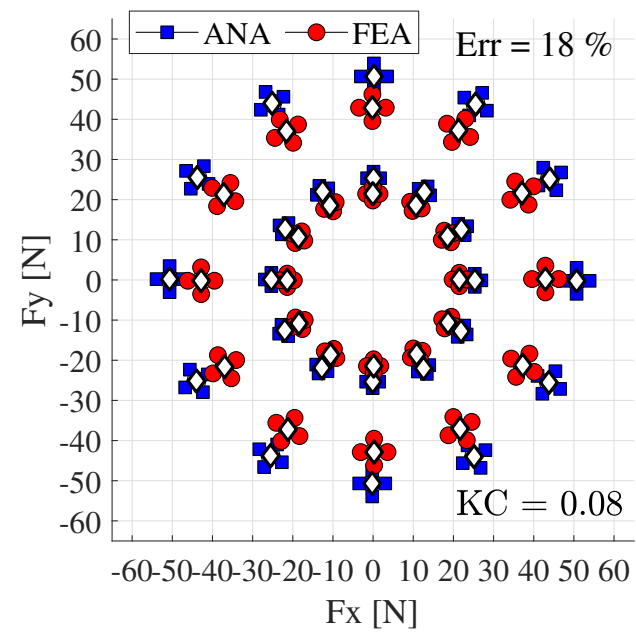

Fig. 5: Different advance angle of the space current $p=2$ and same amplitude. 


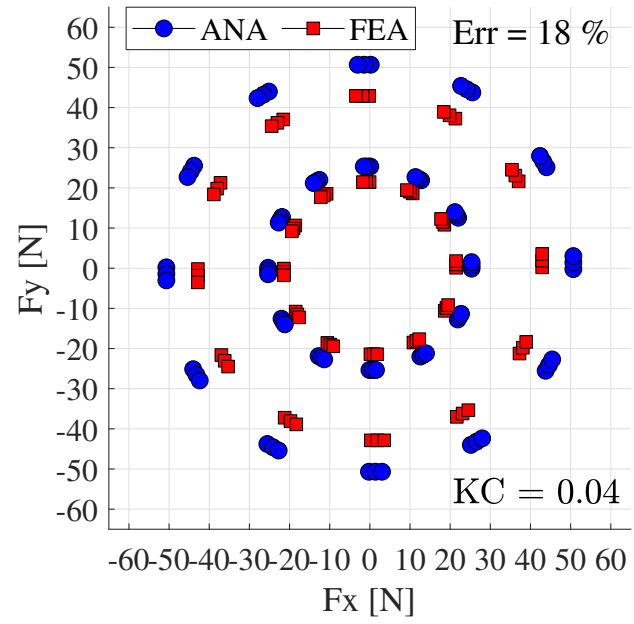

Fig. 6: Different amplitude of the space current $p=2$ and same advance angle 90 [deg].

The variation of the force due to the interaction with the torque draws a cluster of points in the $\mathrm{x}-\mathrm{y}$ plane around the force value generated when zero torque is requested (black diamond marker). In this case the coupling coefficient between torque and force is $K C=0.08$. It is worth to underline that both direction and module of the force change as a consequence of this coupling phenomena and this variation depends on the phase angle of the current vector responsible of the torque production.

A similar analysis is repeated in Fig.6, where different amplitudes of $i_{p 2}$ featuring same advance angle $\left(\angle i_{p 2}=90\right.$ [deg]) are explored. In this case, the coupling effect between torque and force is manifested with a shift in the direction of the force and its value is $K C=0.04$. This analysis is more significant respect with the previous one as it representative of most of the real operating conditions (lying in the constant torque region of the torque-speed characteristic). It is worth to notice that, although some variation in predicting the forces are exhibited by the analytical results, most of the phenomena happening in the motor when both force and torque are produced are fairly replicated by the model although the tangential component of airgap flux density is neglected.

\section{DESIGN CONSIDERATIONS}

Standard design approach for SPM motors tends to size stator teeth and stator/rotor back irons considering only the no-load magnetic scenario when the airgap flux density is generated only by the permanent magnets: $B_{g}=B_{g, 0}$.

In fact, in most of the cases SPM motors meant to generate only electromagnetic torque does not exhibit major variation of the airgap flux density when they operate at the nominal load condition. Indeed, the incremental airgap flux density $B_{g, I}$ dependent by the currents is controlled to stand in quadrature with respect to $B_{g, 0}$.

However, the design of an electric motor done with aim of producing torque and radial forces requires a different approach. In fact, in application where both torque and radial forces are to be generated, the force needs to be produced in any direction and for any rotor position. This condition implies that the incremental airgap flux density $B_{g, i}$ necessary to produce the force and the no load flux density $B_{g, 0}$ can be in phase for certain force directions and rotor positions. Consequently, if the electrical machine is magnetically sized considering only the no-load operating scenario, then when producing radial forces, the soft magnetic material utilization drastically deviates from the designed value. In other words, the electrical machine would experience a much higher saturation when producing radial forces respect to the no load condition. In order to avoid this undesired behaviour, the electrical machine producing both torque and force have to be designed considering the load magnetic scenario.

An another consideration is that the harmonic of flux density $B_{g, i}$ to generate force for this case study is $p=1$, thus requiring for the same amplitude bigger back iron with respect to a $p=2$ flux density waveform.

Considering all the above, Fig.7a presents three designs of the same SPM motor (same airgap radius and $B_{g, 0}$ ) meant to produce the same torque and three different force $(0,5$ and 10 times the weight of the rotor). It is evident how the different $B_{g, i}$ amplitude required for generating the 3 force values determine a significant impact on the stator teeth and back iron dimensions. Another consideration consist in the slot surface increment needed to constrain the current density within the considered limit $\left(3\left[\mathrm{~A} / \mathrm{mm}^{2}\right]\right)$. In fact, motor design that generates higher radial forces requires also higher currents.

Another important aspect to be considered, already highlighted in Sec.III-C, consists in designing the motor in such way that the coupling between torque and forces is reduced. The permanent magnet thickness and the coil pitch are the most important parameters affecting this coupling phenomenon. In order to highlight the impact upon the performance indexes $(K C, K T, K F)$, different magnet thickness (i.e. the magnetic load) and coil pitch are investigated using the numerical implementation of the analytical model. The indexes $K F$ and $K T$ are defined as reported below

$$
\begin{gathered}
K F=\frac{\sqrt{\left(F_{x, a v g}^{2}+F_{y, a v g}^{2}\right)}}{\left|\dot{i_{p 1}}\right|} \\
K T=\frac{T_{a v g}}{\left|\dot{i_{p 2}}\right|}
\end{gathered}
$$

and are the force and torque constant, respectively.

The coil pitch of the winding is changed from 4 to 13 slots pitch while the magnet thickness is varied from $0.5[\mathrm{~mm}]$ to $4.5[\mathrm{~mm}]$. For each combination of these two parameters, the variation of $K C, K T, K F$ are calculated and plotted in Fig. $7 b, c, d$ and e.

In Fig. $7 \mathrm{c}$ and d the capability of producing torque $\left(K_{F}\right)$ and force $\left(K_{F}\right)$ are compared. This pictures show that there is not a unique combination of coil pitch and magnet thickness that maximize both the performance indexes. In fact, the capability of producing torque is enhanced by a coil pitch equal to 6 

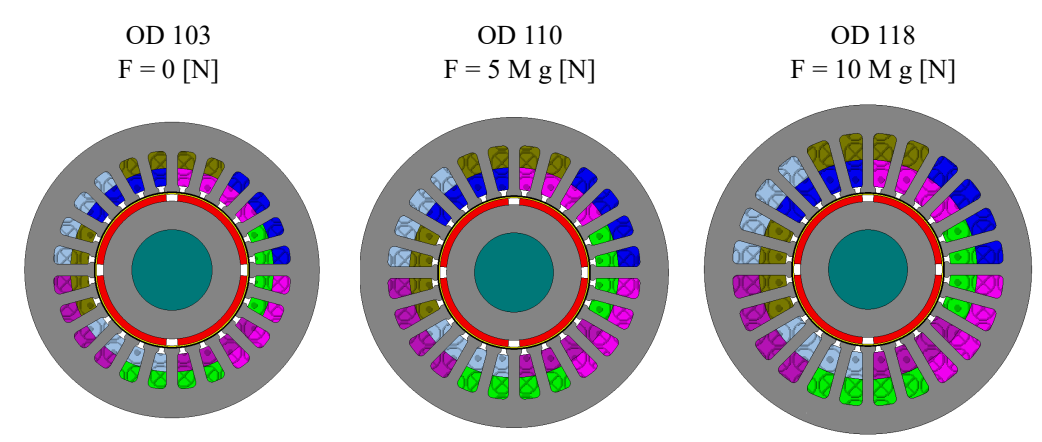

(a) Motors design for different force requirement ( $\mathrm{M}$ is the rotor mass).

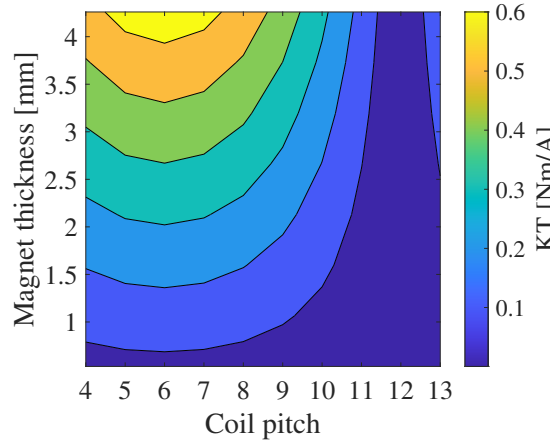

(c) Torque coefficient.

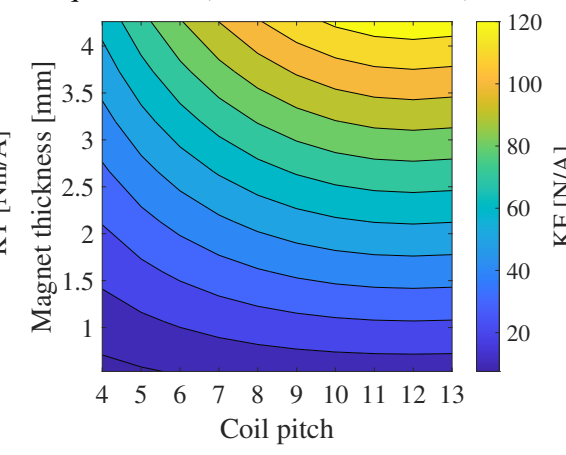

(d) Force coefficient.
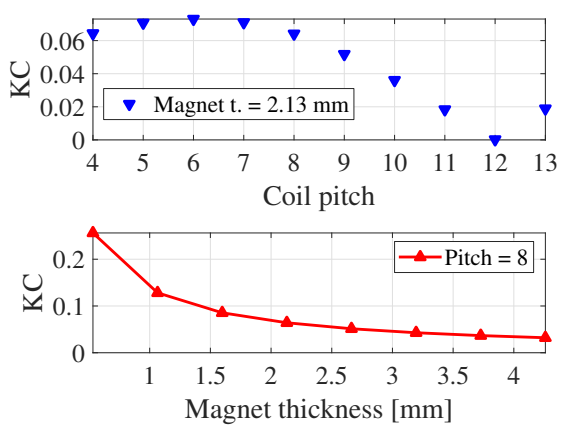

(b) Coupling factor.

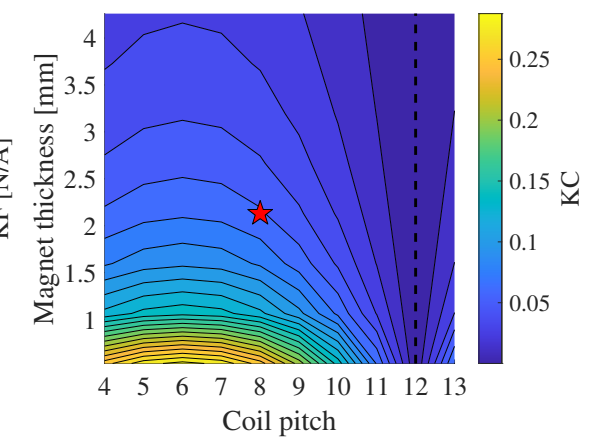

(e) Coupling factor.

Fig. 7: Influence of coil pitch and magnet thickness upon the performance indexes $K C, K T$ and $K F$.

slots while the capability of producing torque is maximized for pitch value close to 12 slots.

However, Fig.7e shows that higher magnet thickness permits to reduce the coupling effect $K C$ between torque and force. The coil pitch of 6 slots exhibits the highest impact upon the coupling coefficient as for the same amplitude of $i_{p 2}$ higher value of torque are achieved. $K C$ drop when the coil pitch is moving toward 12 slots. At 12 slots $K C$ is zero as the motor do not produce any torque due to the zero amplitude of the $p=2$ harmonic. In Fig.7c), the red star represents a good compromise between magnet thickness $(2.13[\mathrm{~mm}])$ and coil pitch (8 slots). This choice is reported in Fig.7b, where one of the two parameter is varied to highlight the $K_{C}$ variations.

On one hand, for the selected coil pitch of 8 slots, the $K C$ value drops as the magnet thickness increase. The decrement is far from being linear and it reaches a plateau for value close to $2[\mathrm{~mm}]$, meaning that more magnet above 2.13 do not bring any significant benefits in term of coupling reduction. On the other hand, for magnet thickness of 2.13 [mm] and a slot pitch 8 represents a good compromise to satisfy force and torque requirements.

\section{CONCLUSION}

This paper has first given an overview of the mathematics behind the electrical machines producing both torque and radial forces. The conditions to be fulfilled in order to produce controllable radial forces have been derived clearly stating the assumption and approximations of the analytical formulation. The numerical implementation of the proposed analytical model has been validated comparing its force and torque predictions with the FE results over a wide range of operating conditions. The analytical model has then been used in order to assess the coupling phenomena between torque and force productions. It has been showed that both amplitude and direction of the force are affected by both amplitude and phase of the current vector responsible for the torque production. The same analytical model has been used to derive several design considerations of multi-Phase bearingless permanent magnet synchronous machine. In fact, the machines designed have increasing force requirement showing how the latter affect the optimal stator geometry. Capability of producing both torque and force has been calculated as function of the magnet thickness and coil pitch along with the coupling coefficient. The trade-off among the need of maximizing both torque and force production along with the need of minimizing the coupling effect have all been carefully discussed.

\section{ACKNOWLEDGMENT}

Another thanks to Rolls-Royce plc and the EPSRC for the support under the Prosperity Partnership Grant Cornerstone: Mechanical Engineering Science to Enable Aero Propulsion Futures“, Grant Ref: EP/R004951/1.

\section{REFERENCES}

[1] X. Sun, L. Chen, and Z. Yang, "Overview of bearingless permanentmagnet synchronous motors," IEEE Transactions on Industrial Electronics, vol. 60, no. 12, pp. 5528-5538, 2013.

[2] A. Chiba, T. Fukao, O. Ichikawa, M. Oshima, M. Takemoto, and D. Dorrell, Magnetic Bearings and Bearingless Drives. Elsevier Science, 2005. 
[3] W. K. S. Khoo, K. Kalita, and S. D. Garvey, "Practical implementation of the bridge configured winding for producing controllable transverse forces in electrical machines," IEEE Transactions on Magnetics, vol. 47, no. 6, pp. 1712-1718, 2011.

[4] E. Severson, S. Gandikota, and N. Mohan, "Practical implementation of dual-purpose no-voltage drives for bearingless motors," IEEE Transactions on Industry Applications, vol. 52, no. 2, pp. 1509-1518, 2016.

[5] J. Huang, B. Li, H. Jiang, and M. Kang, "Analysis and control of multiphase permanent-magnet bearingless motor with a single set of halfcoiled winding," IEEE Transactions on Industrial Electronics, vol. 61, no. 7, pp. 3137-3145, 2014.

[6] G. Valente, A. Formentini, L. Papini, C. Gerada, and P. Zanchetta, "Performance improvement of bearingless multisector pmsm with optimal robust position control," IEEE Transactions on Power Electronics, vol. 34, no. 4, pp. 3575-3585, 2019.

[7] G. Sala, G. Valente, M. D. Nardo, M. Degano, P. Zanchetta, and C. Gerada, "Power-sharing control in bearingless multi-sector and multi-threephase permanent magnet machines," IEEE Transactions on Industrial Electronics, vol. 68, no. 10, pp. 9070-9080, 2021.

[8] G. Sala, G. Valente, A. Formentini, L. Papini, D. Gerada, P. Zanchetta, A. Tani, and C. Gerada, "Space vectors and pseudoinverse matrix methods for the radial force control in bearingless multisector permanent magnet machines," IEEE Transactions on Industrial Electronics, vol. 65, no. 9, pp. 6912-6922, 2018.

[9] S. Serri, A. Tani, and G. Serra, "Analytical model of radial forces considering mutual effects between torque and levitation current space vectors in 5-phase pm bearingless motors," in IECON 2013 - 39th Annual Conference of the IEEE Industrial Electronics Society, 2013, pp. 5142-5147.

[10] - "A method for non-linear analysis and calculation of torque and radial forces in permanent magnet multiphase bearingless motors," in International Symposium on Power Electronics Power Electronics, Electrical Drives, Automation and Motion, 2012, pp. 75-82.

[11] P. Bolognesi, "A mid-complexity analysis of long-drum-type electric machines suitable for circuital modeling," in 2008 18th International Conference on Electrical Machines, 2008, pp. 1-5.

[12] A. Tessarolo, L. Branz, and M. Bortolozzi, "Stator inductance matrix diagonalization algorithms for different multi-phase winding schemes of round-rotor electric machines part ii." in IEEE EUROCON 2015 International Conference on Computer as a Tool (EUROCON).

[13] A. Marfoli, L. Papini, P. Bolognesi, and C. Gerada, "An analyticalnumerical approach to model and analyse squirrel cage induction motors," IEEE Transactions on Energy Conversion, vol. 36, no. 1, pp. 421430,2021 